\title{
HAMILTONIAN CYCLES IN CERTAIN GRAPHS
}

\author{
KATHERINE HEINRICH and W. D. WALLIS
}

(Received 3 August; revised 7 December 1977)

Communicated by T. E. Hall

\begin{abstract}
It is observed that arrays which arise in the scheduling of tournaments exist if and only if there are Hamiltonian cycles in certain graphs. The graphs are generalizations of those which arise in the "Footballers of Croam" problem. It is proven that such Hamiltonian cycles exist in infinite classes of the graphs.
\end{abstract}

Subject classification (Amer. Math. Soc. (MOS) 1970): 05 C 35, 05 B 30.

\section{Introduction}

We consider the following problem. Given positive integers $m, n$ and $g$, is it possible to arrange all the subsets of size $m$ of an $n$-set $S$ in an array with $g$ columns such that

(i) each subset appears exactly once in the array;

(ii) there are exactly $g$ subsets in each row, except possibly the last;

(iii) no element of $S$ appears more than once in any row;

(iv) no element of $S$ appears in two successive rows;

(v) no element of $S$ appears in both the first and the last row.

Grant (1975) pointed out that arrays with these properties (and similar arrays with property (v) relaxed) arise in the scheduling of tournaments; the interested reader should see his paper for details. Grant studied the case $m=2$, for arbitrary $n$ and $g$.

The arrays are also related to a well-known problem of graph theory. Let $G_{n, m}$ denote the graph whose vertices are labelled with the $m$-subsets of an $n$-set, two vertices being adjacent if and only if their inducing sets are disjoint. Then $G_{n, m}$ has $\left(\begin{array}{l}n \\ m\end{array}\right)$ vertices and $\frac{1}{2}\left(\begin{array}{l}n \\ m\end{array}\right)\left(\begin{array}{c}n-m \\ m\end{array}\right)$ edges. An array satisfying the five conditions 
of the problem in the case $g=1$ exists if and only if $G_{n, m}$ has a Hamiltonian cycle.

The case $n=2 k-1, m=k-1, g=1$ has been considered by several authors. Meredith and Lloyd (1972) called $G_{2 k-1, k-1}$ "the graph $0_{k}$ " and conjectured that, for $k \geqslant 4,0_{k}$ is an edge-disjoint union of Hamiltonian cycles plus, perhaps, a 1-factor. It is easily verified that $\mathrm{O}_{2}$ has a Hamiltonian cycle and that $\mathrm{O}_{3}$ does not $\left(0_{3}\right.$ is in fact the Petersen graph). The graphs $0_{4}, 0_{5}, 0_{6}, 0_{7}$ and $0_{8}$ have all been shown to have Hamiltonian cycles (see Balaban, 1972; Meredith and Lloyd, 1973; Mather, 1976). The problem appears to be very difficult.

For the rest of the paper we restrict our attention to the "graphical" case $g=1$, but consider general $m$ and $n$ with $n \geqslant 2 m+1$. (If $n \leqslant 2 m$ the array is impossible and, in fact, the graph is disconnected, unless $m=1$.) Without loss of generality we may choose the $n$-set $S$ to be $\{1,2, \ldots, n\}$.

The authors wish to thank Mr. Ross Dunstan, who found the proof of Lemma 4.3.1, and also wish to thank the referee for helpful comments.

\section{Graph-theoretic preliminaries}

We shall use two standard results of graph theory-Phillip Hall's Theorem on matchings and Ore's Theorem on the existence of Hamiltonian cycles.

If $G$ is a bipartite graph on two sets $X$ and $Y$, written as $G=G(X, Y)$, then a complete matching of $X$ into $Y$ is a set of edges such that no two edges are incident and every vertex of $X$ appears in some edge. The following theorem is due to P. Hall (1935).

THEOREM 2.1. Let $G=G(X, Y)$ be a bipartite graph. There exists a complete matching of $X$ into $Y$ if and only if for all $A \subseteq X,|A| \leqslant|R(A)|$ where $R(A)$ is the set of vertices in $Y$ adjacent to vertices of $A$.

COROLlARY 2.1.1. In a bipartite graph $G(X, Y)$ there exists a complete matching of $X$ into $Y$ if every vertex in $X$ is adjacent to $k$ or more vertices in $Y$ and every vertex in $Y$ is adjacent to $k$ or fewer vertices in $X$.

The proof of the corollary follows easily from the theorem.

THEOREM 2.2 (Ore, 1960). A graph with $p \geqslant 3$ vertices has a Hamiltonian cycle if, for every pair $u$ and $v$ of non-adjacent vertices,

$$
\text { degree } u+\text { degree } v \geqslant p \text {. }
$$

COROLLARY 2.2.1. $G_{n, m}$ has a Hamiltonian cycle whenever $2\left(\begin{array}{c}n-m \\ m\end{array}\right) \geqslant\left(\begin{array}{c}n \\ m\end{array}\right)$. 
3. The cases $m=1,2,3$

The existence of a Hamiltonian cycle in $G_{n, m}$ is easy to discuss when $m=1$ or 2 . The case $m=3$ is more difficult and takes up most of this section.

It is interesting to notice that $G_{n, 1}$ is the complete graph $K_{n}$, and $G_{n, 2}$ is the complement of the line graph of $K_{n}$.

THEOREM 3.1. The graph $G_{n, 1}, n \geqslant 3$, has a Hamiltonian cycle.

The proof is trivial.

THEOREM 3.2. The graph $G_{n, 2}, n \geqslant 6$, has a Hamiltonian cycle.

Proof. The graph $G_{n, 2}$ has $\left(\begin{array}{l}n \\ 2\end{array}\right)$ vertices and is regular of degree $\left(\begin{array}{c}n-2 \\ 2\end{array}\right)$. As $2\left(\begin{array}{c}n-2 \\ 2\end{array}\right) \geqslant\left(\begin{array}{l}n \\ 2\end{array}\right)$ for $n \geqslant 8$, then by Corollary $2.2 .1, G_{n, 2}, n \geqslant 8$, always has a Hamiltonian cycle. The following sequences of vertices illustrate Hamiltonian cycles in $G_{6,2}$ and $G_{7,2}$ respectively:

$25,16,23,45,12,34,56,13,24,35,46,15,26,14,36$;

$25,37,12,34,56,17,23,45,67,13,24,35,46,57,16,27,36,47,15,26,14$.

THEOREM 3.3. The graph $G_{n, 3}, n \geqslant 7$, has a Hamiltonian cycle.

Proof. Theorem 2.2 implies that $G_{n, 3}$ has a Hamiltonian cycle for $n \geqslant 16$ and Theorem 4.2 will prove that $G_{n, 3}$ has a Hamiltonian cycle when $n=12$, 13 or 15 leaving only the cases $n=7,8,9,10,11$ or 14 ; however, it is shorter to give a general proof than to exhibit the six Hamiltonian cycles.

The proof is by induction. We begin by exhibiting sequences of vertices which are Hamiltonian cycles in $G_{7,3}$ (due to Balaban, 1972) and in $G_{8,3}$, respectively:

$236,157,246,135,467,125,346,257,134,256,137,456,123,567,234$, $167,235,147,356,127,345,267,145,237,146,357,124,367,245,136$, $247,156,347,126,457$;

$247,138,567,348,156,234,678,124,356,178,235,146,578,134,256$, $347,568,137,248,357,468,157,236,145,268,147,368,257,168,237$, $458,127,346,125,378,245,136,278,456,123,478,126,345,128,457$, $238,167,258,367,148,267,135,246,158,467,358$. 
Now assume that the graph $G_{n-2,3}$ has a Hamiltonian cycle. Also assume that $n \geqslant 9$.

Writing $V$ for the vertex set of $G_{n, 3}$, partition $V$ into four subsets, $V_{1}, V_{2}, V_{3}$ and $V_{4}$ as follows: $V_{1}$ consists of all vertices whose indexing sets contain neither of the elements 1 and $2 ; V_{2}$ consists of all vertices whose indexing sets contain 1 but not 2; $V_{3}$ has all vertices whose indexing sets contain 2 but not 1 ; and $V_{4}$ has all vertices whose indexing sets contain both 1 and 2. Obviously $V=V_{1} \cup V_{2} \cup V_{3} \cup V_{4}$ and $\left|V_{1}\right|=\left(\begin{array}{c}n-2 \\ 3\end{array}\right),\left|V_{2}\right|=\left|V_{3}\right|=\left(\begin{array}{c}n-2 \\ 2\end{array}\right)$ and $\left|V_{2}\right|=\left(\begin{array}{c}n-2 \\ 1\end{array}\right)$. By the induction hypothesis the subgraph induced by $V_{1}$ has a Hamiltonian cycle; we denote it by $C$.

We now consider inserting the vertices of $V_{4}$ into $C$ in the following way. Say $v=v_{1} v_{2} v_{3}$ and $u=u_{1} u_{2} u_{3}$ are two adjacent vertices in $C$ and $w=12 w_{1}$ is a vertex of $V_{4}$ where $w_{1} \notin\left\{v_{1}, v_{2}, v_{3}, u_{1}, u_{2}, u_{3}\right\}$. Then we can extend $C$ to include $w$ by deleting the edge $(u, v)$ and adding the edges $(u, w)$ and $(w, v)$. For any edge of $C$ there are $n-8$ possible vertices in $V_{4}$ that could be inserted into this edge. So with each of the $\left(\begin{array}{c}n-2 \\ 3\end{array}\right)$ edges of $C$ we can associate a set of $n-8$ vertices of $V_{4}$.

We next count the number of these sets which contain a given vertex of $V_{4}$. Consider the vertex $u=12 u_{1}$ of $V_{4}$. As $u_{1}$ appears in the indexing set of $\left(\begin{array}{c}n-3 \\ 2\end{array}\right)$ vertices of $V_{1}$, none of which are adjacent, then $u$ is contained in

$$
\left(\frac{n-2}{3}\right)-2\left(\frac{n-3}{2}\right)=(n-8)(n-4)(n-3) / 6
$$

of the sets associated with the edges of $C$; and as $n \geqslant 9$ each vertex of $V_{4}$ is contained in at least $5(n-8)$ of these sets.

Construct a bipartite graph $G=G(X, Y)$ where $X$ is the set of vertices 'of $V_{4}$ and $Y$ is the set of edges in $C$. If $x \in X$ and $y \in Y$ then $x$ is adjacent to $y$ if and only if $x$ is contained in the set associated with the edge $y$. Thus each vertex in $X$ has degree $(n-8)(n-4)(n-3) / 6 \geqslant 5(n-8)$, as $n \geqslant 9$, and each vertex of $Y$ has degree $n-8$. Putting $k=n-8$ we can, using Corollary 2.1 , associate each vertex of $V_{4}$ with a distinct edge of $C$. Therefore the cycle $C$ can be extended to a cycle $C^{\prime}$ which passes through all vertices of $V_{1} \cup V_{4}$.

We consider separately the cases when $\left(\begin{array}{c}n-2 \\ 2\end{array}\right)$ is odd and when $\left(\begin{array}{c}n-2 \\ 2\end{array}\right)$ is even.

Case 1 . When $\left(\begin{array}{c}n-2 \\ 2\end{array}\right)$ is odd. Consider the vertex sets $V_{2}$ and $V_{3}$. In $V_{2}$ delete the element 1 from all indexing sets and draw the graph $G_{n-2,2}$ on this new set of vertices, $V_{2}^{\prime}$. Similarly delete 2 from $V_{3}$ and draw the graph $G_{n-2,2}$ on $V^{\prime}$. Since 
by Theorem 3.2, $G_{n-2,2}$ has a Hamiltonian cycle for $n-2 \geqslant 6$ we can draw Hamiltonian cycles through the vertices of $V_{2}^{\prime}$ and $V_{3}^{\prime}$. Permute the elements of the indexing sets in these cycles so that we have $u=34$ adjacent to $v=56$ and then delete the edges $(u, v)$ from both cycles to obtain two Hamiltonian paths of odd length; their lengths are $\left(\begin{array}{c}n-2 \\ 2\end{array}\right)$. In $V_{2}^{\prime}$ relabel $u_{1}=34$ with $u_{1}^{\prime}=134$. Then if $w=w_{1} w_{2}$ is adjacent to $u$ relabel it with $w^{\prime}=2 w_{1} w_{2}$. Continue in this manner until $v_{1}=56$ is relabelled with $v_{1}^{\prime}=156$ (as $\left(\begin{array}{c}n-2 \\ 2\end{array}\right)$ is odd). For $V_{3}^{\prime}$ relabel $u_{2}=34$ with $u_{2}^{\prime}=234$ and continue as for $V_{2}^{\prime}$ until $v_{2}=56$ is relabelled $v_{2}^{\prime}=256$. Then draw an edge joining the vertices $u_{1}^{\prime}=134$ and $v_{2}^{\prime}=256$. We now have a Hamiltonian path through the vertices of $V_{2} \cup V_{3}$.

We return now to the Hamiltonian cycle $C^{\prime}$ through the vertices of $V_{1} \cup V_{4}$. Here there must be two adjacent vertices neither of which has the elements 1 and 2 in its indexing set as otherwise we have $n-2 \geqslant\left(\begin{array}{c}n-2 \\ 3\end{array}\right)$ which is never true for $n \geqslant 9$. Permute the elements of the indexing sets in $V_{1} \cup V_{4}$ so that vertex $u_{3}=347$ is adjacent to $v_{3}=568$. We can then draw edges from $u_{2}^{\prime}=234$ to $v_{3}=568$ and from $v_{2}^{\prime}=256$ to $u_{3}=347$ and we now have a Hamiltonian cycle in $G_{n, 3}, n \geqslant 9$, $\left(\begin{array}{c}n-2 \\ 2\end{array}\right)$ odd.

Case 2. When $\left(\begin{array}{c}n-2 \\ 2\end{array}\right)$ is even. Note that we now only require $n \geqslant 10$ as $\left(\begin{array}{c}9-2 \\ 2\end{array}\right)$ is odd. Construct the two paths through the vertices of $V_{2} \cup V_{3}$ as was done in Case 1. However, this time the endpoints of one path, $P_{1}$, are $u_{1}=234, v_{1}=156$ and of the other, $P_{2}$, are $u_{2}=134, v_{2}=256$. Again, relabel the Hamiltonian cycle $C^{\prime}$ through $V_{1} \cup V_{4}$ so that $u_{3}=347$ and $v_{3}=568$ are adjacent. We now have a cycle and two paths which we wish to join together to form a Hamiltonian cycle in $G_{n, 3}, n \geqslant 10,\left(\begin{array}{c}n-2 \\ 2\end{array}\right)$ even. First add the edge $\left(u_{2}, v_{2}\right)$. The number of vertices in $P_{2}$ with 1 in their indexing sets is $\frac{1}{2}\left(\begin{array}{c}n-2 \\ 2\end{array}\right)$ whilst the number with either 3 or 4 in their indexing sets is $2\left(\begin{array}{c}n-4 \\ 1\end{array}\right)+1$. As $\frac{1}{2}\left(\begin{array}{c}n-2 \\ 2\end{array}\right)>2\left(\begin{array}{c}n-4 \\ 1\end{array}\right)+1$ for $n \geqslant 10$ then clearly we have in $P_{2}$ a vertex $v=1 x_{1} y_{1}$ where neither $x_{1}$ nor $y_{1}$ is 3 or 4 . Let $u=2 x_{2} y_{2}, u \neq 256$, be the vertex adjacent to $v$ in $P_{2}$. Delete the edge $(u, v)$ and add the edges $\left(v_{1}, u_{3}\right)$ and $\left(v, u_{1}\right)$. The indexing set of $u$ can contain at most two of 5,6 and 8 . If it contains any of these make the relevant exchange of the elements $x_{2}$ and 9 or $y_{2}$ and 10 in $C^{\prime}$; then add the edge $\left(u, v_{3}\right)$. On deleting $\left(u_{3}, v_{3}\right)$ we have a Hamiltonian cycle in $G_{n, 3}, n \geqslant 10,\left(\begin{array}{c}n-2 \\ 2\end{array}\right)$ even. 
Therefore, combining the two cases, we have that the graph $G_{n, 3}, n \geqslant 7$, always contains a Hamiltonian cycle.

\section{Cases where $n$ is large relative to $m$}

In this section we derive two theorems which state that $G_{n, m}$ has a Hamiltonian cycle in cases were $n$ is sufficiently large relative to $m$. Theorem 4.2 uses a result on block designs. A balanced incomplete block design is said to be resolvable if its blocks can be partitioned into sets in such a way that every set contains each treatment precisely once. (The reader is referred to Chapters 7, 8 and 10 of Street and Wallis, 1977.)

THEOREM 4.1 (Baranyai, 1975). The balanced incomplete block design with parameters $\left(k m,\left(\begin{array}{c}k m \\ m\end{array}\right),\left(\begin{array}{c}k m-1 \\ m-1\end{array}\right), m,\left(\begin{array}{c}k m-2 \\ m-2\end{array}\right)\right)$ in which the blocks are all the $m$-sets of a set of $\mathrm{km}$ treatments is resolvable.

We can now proceed to the lemmas needed for the proof of the main result.

LeMma 4.2.1. Given an $n$-set $S$ let $X$ be the set of all $r$-sets of $S$ and $Y$ the set of all $l$-sets of $S, n \geqslant l+r$ and $l>r$. We can associate with each $r$-set an $l$-set which contains the r-set, that is, if the r-set is $x_{1} x_{2} \ldots x_{r}$ then the associated $l$-set is $x_{1} x_{2} \ldots x_{r} x_{r+1} \ldots x_{l}$; so that different $l$-sets are associated with different $r$-sets.

Proof. Construct a bipartite graph $G=G(X, Y)$ in the following way. Let the vertices of $X$ be the $r$-sets of $S$ and the vertices of $Y$ be the $l$-sets of $S$, and let vertices be adjacent if one contains the other. Then each vertex in $X$ has degree $\left(\begin{array}{l}n-r \\ l-r\end{array}\right)$ and each vertex in $Y$ has degree $\left(\begin{array}{l}l \\ r\end{array}\right)$. So if $\left(\begin{array}{l}n-r \\ l-r\end{array}\right) \geqslant\left(\begin{array}{l}l \\ r\end{array}\right)$ then by Corollary 2.1.1 there is a complete matching of $X$ into $Y$ and hence the lemma is proven.

If $n \geqslant l+r, l>r$, then $r<\frac{1}{2} n$. Now either $n-l$ or $l$ is $\leqslant \frac{1}{2} n$ and both are bigger than or equal to $r$ so either $\left(\begin{array}{c}n \\ n-l\end{array}\right) \geqslant\left(\begin{array}{l}n \\ r\end{array}\right)$ or $\left(\begin{array}{l}n \\ l\end{array}\right) \geqslant\left(\begin{array}{l}n \\ r\end{array}\right)$. But both these statements are the same. Hence $(n-r) ! r ! \geqslant(n-l) ! l !$ and so $\left(\begin{array}{l}n-r \\ l-r\end{array}\right) \geqslant\left(\begin{array}{l}l \\ r\end{array}\right)$.

LEMMA 4.2.2. The graph $G_{n, m}, n=k m$, always has a Hamiltonian cycle when $k \geqslant m+1$.

Proof. The vertices of $G_{n, m}$ are labelled with the blocks of the balanced incomplete block design of Theorem 4.1. Therefore, we can partition the vertex set 
of $G_{n, m}$ into $p=\left(\begin{array}{c}k m-1 \\ m-1\end{array}\right)$ classes $R_{1}, R_{2}, \ldots, R_{p}$ each of size $k$. It is easy to see that the subgraph induced by any one of these $p$ classes will be the complete graph on $k$ vertices, $K_{k}$. In $R_{i}$ we have a vertex $v_{i}$ labelled with the indexing set $1 x_{1}^{i} x_{2}^{i} \ldots x_{m-1}^{i}$, for all $i \in\{1,2, \ldots, p\}$. As at most $m$ of the vertices of $R_{i+1}$ can contain elements of the indexing set of $v_{i}$ and as there are $k \geqslant m+1$ vertices in $R_{i+1}$ then there is at least one vertex $u_{i+1}$ whose indexing set is disjoint from that of $v_{i}$. Therefore, we can draw an edge from $v_{i}$ to $u_{i+1}$. Continue in this manner joining $R_{i}$ to $R_{i+1}$ finally joining $R_{p}$ to $R_{1}$. Obviously, as $u_{i}$ and $v_{i}$ are distinct and both are vertices in a complete graph $K_{k}$, there is a Hamiltonian path in $K_{k}$ of which they are the endpoints. The Hamiltonian cycle $C$ in $G_{n, m}, n=k m, k \geqslant m+1$, can now clearly be seen.

THEOREM 4.2. The graph $G_{n, m}$ where $n=k m+l, 0 \leqslant l<m$, will always have a Hamiltonian cycle if $k \geqslant \max \left\{2^{l+1}, m+1\right\}$.

Proof. Assume that $k \geqslant \max \left\{2^{l+1}, m+1\right\}$ where $n=k m+l, 0 \leqslant l<m$. In the case $l=0$ we have $k \geqslant \max \{2, m+1\}$ and as $m \geqslant 1$ then always $k \geqslant m+1$ and by Lemma 4.2.2, $G_{n, m}, n=k m$, always has a Hamiltonian cycle. Thus we can assume $0<l<m$.

Let $S$ be the $n$-set from which the vertices of $G_{n, m}, n=k m+l$, are labelled. Select a subset of $l$ elements from $S$, say $Y=\left\{y_{1}, y_{2}, \ldots, y_{l}\right\}$, and use the remaining $k m$ elements to construct $G^{\prime}=G_{k m, m}$. Draw the Hamiltonian cycle $C$ in $G^{\prime}$ as constructed in Lemma 4.2.2.

We want to insert the remaining vertices of $G_{n, m}$ into the edges of $C$, as was done in the first part of Theorem 3.3.

Before we begin some notation is needed. Write $\left[\left(\begin{array}{l}l \\ r\end{array}\right)\right]\left[\left(\begin{array}{c}k m \\ m-r\end{array}\right)\right]$ to indicate an $m$-set of $S$ of the form $y_{1} y_{2} \ldots y_{r} x_{1} x_{2} \ldots x_{m-r}$ where $y_{i} \in Y, x_{i} \in S \backslash\{Y \cup\{1\}\}$, and $1\left[\left(\begin{array}{l}l \\ r\end{array}\right)\right]\left[\left(\begin{array}{c}k m \\ m-r-1\end{array}\right)\right]$ to indicate an $m$-set $1 y_{1} y_{2} \ldots y_{r} x_{1} x_{2} \ldots x_{m-r-1}$ where $y_{i} \in Y$, $x_{i} \in S \backslash\{Y \cup\{1\}\}$. When we speak of $u_{i}, v_{i}$ and $R_{i}$ they are the $u_{i}, v_{i}$ and $R_{i}$ of Lemma 4.2.2.

Thus the vertices of $G_{n, m}$, not in $G^{\prime}$, are labelled

$$
\begin{aligned}
& {\left[\left(\begin{array}{l}
l \\
1
\end{array}\right)\right]\left[\left(\begin{array}{c}
k m \\
m-1
\end{array}\right)\right], 1\left[\left(\begin{array}{l}
l \\
1
\end{array}\right)\right]\left(\left[\begin{array}{c}
k m \\
m-2
\end{array}\right)\right],\left[\left(\begin{array}{l}
l \\
2
\end{array}\right)\right]\left[\left(\begin{array}{c}
k m \\
m-2
\end{array}\right)\right], 1\left[\left(\begin{array}{l}
l \\
2
\end{array}\right)\right]\left[\left(\begin{array}{c}
k m \\
m-3
\end{array}\right)\right], \ldots,} \\
& {\left[\left(\begin{array}{c}
l \\
l-1
\end{array}\right)\right]\left[\left(\begin{array}{c}
k m \\
m-l+1
\end{array}\right)\right], 1\left[\left(\begin{array}{c}
l \\
l-1
\end{array}\right)\right]\left[\left(\begin{array}{c}
k m \\
m-l
\end{array}\right)\right],\left[\left(\begin{array}{l}
l \\
l
\end{array}\right)\right]\left[\left(\begin{array}{c}
k m \\
m-l
\end{array}\right)\right], 1\left[\left(\begin{array}{l}
l \\
l
\end{array}\right]\right)\left[\left(\begin{array}{c}
k m \\
m-l-1
\end{array}\right)\right] .}
\end{aligned}
$$

Insert these vertices into the Hamiltonian cycle $C$ of $G^{\prime}$ in the following way. 
With each $v_{i}$ in $C$ we can associate $\left(\begin{array}{l}l \\ 1\end{array}\right)$ vertices of the form $\left[\left(\begin{array}{l}l \\ 1\end{array}\right)\right]\left[\left(\begin{array}{c}k m \\ m-1\end{array}\right)\right]$, where if $v_{i}=1 x_{1} x_{2} \ldots x_{m-1}$ then $\left[\left(\begin{array}{c}k m \\ m-1\end{array}\right)\right]=x_{1} x_{2} \ldots x_{m-1}$. Consider the path of $C$ in $R_{i}$ from $v_{i}$ to $u_{i}$. Since $x_{j}$ does not occur in the indexing set of any vertex in this path except for $v_{i}$ then we can extend each edge of the path, except for that edge incident with $v_{i}$, to include one of the $\left(\begin{array}{l}l \\ 1\end{array}\right)$ vertices. This can be done provided the path has at least $\left(\begin{array}{l}l \\ 0\end{array}\right)+\left(\begin{array}{l}l \\ 1\end{array}\right)$ edges, i.e. $R_{i}$ has $1+\left(\begin{array}{l}l \\ 0\end{array}\right)+\left(\begin{array}{l}l \\ 1\end{array}\right)$ vertices. And as $k \geqslant \max \left\{2^{l+1}, m+1\right\}$ this is always true.

Take the vertices of the form $1\left[\left(\begin{array}{l}l \\ 1\end{array}\right)\right]\left[\left(\begin{array}{c}k m \\ m-2\end{array}\right)\right]$ and $\left[\left(\begin{array}{l}l \\ 2\end{array}\right)\right]\left[\left(\begin{array}{c}k m \\ m-2\end{array}\right)\right]$. For each $\left[\left(\begin{array}{c}k m \\ m-2\end{array}\right)\right]=x_{1} x_{2} \ldots x_{m-2}$ there is a different $v_{i}, i \in\left\{1,2, \ldots, p=\left(\begin{array}{c}k m-1 \\ m-1\end{array}\right)\right\}$, such that $v_{i}=1 x_{1} x_{2} \ldots x_{m-2} z_{1}$. (This follows from Lemma 4.2.1.) So to extend the path in $R_{i}$ from $v_{i}$ to $u_{i}$ to include these $\left(\begin{array}{l}l \\ 1\end{array}\right)+\left(\begin{array}{l}l \\ 2\end{array}\right)$ vertices $R_{i}$ must have a further $\left(\begin{array}{l}l \\ 1\end{array}\right)+\left(\begin{array}{l}l \\ 2\end{array}\right)$ edges, i.e. $R_{i}$ must have at least $1+\left[\left(\begin{array}{l}l \\ 0\end{array}\right)+\left(\begin{array}{l}l \\ 1\end{array}\right)\right]+\left[\left(\begin{array}{l}l \\ 1\end{array}\right)+\left(\begin{array}{l}l \\ 2\end{array}\right)\right]$ vertices; and there will be this many vertices as $k \geqslant \max \left\{2^{l+1}, m+1\right\}$.

Continue in this manner until finally we have only to add $1\left[\left(\begin{array}{l}l \\ l\end{array}\right)\right]\left[\left(\begin{array}{c}\mathrm{km} \\ m-l-1\end{array}\right)\right]$ where for each $\left[\left(\begin{array}{c}k m \\ m-l-1\end{array}\right)\right]=x_{1} x_{2} \ldots x_{m-l-1}$ there is a different $v_{i}, i \in\{1,2, \ldots, p\}$ such that $v_{i}=1 x_{1} x_{2} \ldots x_{m-l-1} z_{1} z_{2} \ldots z_{l}$ (again by Lemma 4.2.1). Then to extend the path in $R_{i}$ from $v_{i}$ to $u_{i}$ it follows that $R_{i}$ must have a further $\left(\begin{array}{l}l \\ l\end{array}\right)$ edges and so have at least

$$
\begin{aligned}
1+\left[\left(\begin{array}{l}
l \\
0
\end{array}\right)+\left(\begin{array}{l}
l \\
1
\end{array}\right)\right]+\left[\left(\begin{array}{l}
l \\
1
\end{array}\right)+\left(\begin{array}{l}
l \\
2
\end{array}\right)\right] & +\left[\left(\begin{array}{l}
l \\
2
\end{array}\right)+\left(\begin{array}{l}
l \\
3
\end{array}\right)\right]+\ldots+\left[\left(\begin{array}{c}
l \\
l-1
\end{array}\right)+\left(\begin{array}{l}
l \\
l
\end{array}\right)\right]+\left(\begin{array}{l}
l \\
l
\end{array}\right) \\
& =2\left[\left(\begin{array}{l}
l \\
0
\end{array}\right)+\left(\begin{array}{l}
l \\
1
\end{array}\right)+\left(\begin{array}{l}
l \\
2
\end{array}\right)+\ldots+\left(\begin{array}{l}
l \\
l
\end{array}\right)\right]=2^{l+1} \text { vertices. }
\end{aligned}
$$

As $k \geqslant \max \left\{2^{l+1}, m+1\right\}$ this is always possible.

Therefore $G_{n, m}, n=k m+l$, has a Hamiltonian cycle when $k \geqslant \max \left\{2^{l+1}, m+1\right\}$.

Given a positive integer $m$, we shall define $N(m)$ to be the smallest positive integer such that, for all $n \geqslant N(m), 2\left(\begin{array}{c}n-m \\ m\end{array}\right) \geqslant\left(\begin{array}{l}n \\ m\end{array}\right)$. 
Lemma 4.3.1.

$$
\frac{m \cdot \sqrt[m]{2}}{\sqrt[m]{2-1}}-1 \leqslant N(m) \leqslant \frac{m \cdot \sqrt[m]{2}}{\sqrt[m]{2-1}}+m
$$

ProOF. It is easiest to consider the ratio

$$
\begin{aligned}
& M=\left(\frac{n}{m}\right) /\left(\frac{n-m}{m}\right) \\
& M=\left(1-\frac{m}{n}\right)\left(1-\frac{m}{n-1}\right) \ldots\left(1-\frac{m}{n-m+1}\right) .
\end{aligned}
$$

Clearly

$$
\left(1-\frac{m}{n-m+1}\right)^{m} \leqslant M \leqslant\left(1-\frac{m}{n}\right)^{m} \text {. }
$$

Now

$$
\left(1-\frac{m}{n-m+1}\right)^{m} \geqslant \frac{1}{2}
$$

implies $M \geqslant \frac{1}{2}$, and the left-hand inequality is equivalent to

$$
n \geqslant \frac{m \cdot \sqrt[m]{2}}{\sqrt[m]{2-1}}+(m-1)
$$

so the smallest integer not less than this is an upper bound for $N(m)$, and

$$
N(m) \leqslant \frac{m \cdot \sqrt[m]{2}}{\sqrt[m]{2}-1}+m .
$$

If

$$
\left(1-\frac{m}{n}\right)^{m}<\frac{1}{2}
$$

then $M<\frac{1}{2}$ and $n<N(m)$. So

$$
\frac{m \cdot \sqrt[m]{2}}{m \sqrt{2}-1} \leqslant N(m)
$$

From the lemma and Corollary 2.2.1 we have

THEOREM 4.3. If

$$
n \geqslant \frac{m \sqrt{2 . m}}{m \sqrt{2-1}}+m
$$

then $G_{n, m}$ has a Hamiltonian cycle. 
We note that $N(m)$ is asymptotically equal to $m^{2} \log _{2} e$, and approaches this value from above. The relative error in using this approximation is quite small (about $5 \%$ at $m=20$ ).

\section{References}

A. T. Balaban (1972), "Chemical graphs, Part XIII: Combinatorial patters", Rev. Roumaine Math. Pures Appl. 17, 3-16.

Zs. Baranyai (1975), "On the factorization of the complete uniform hypergraph", Infinite and Finite Sets, Vol. 1, pp. 91-108 (North-Holland Publishing Co.).

Douglas D. Grant (1975), "How to run a hockey tournament-a combinatorial scheduling problem", Proc. 5th British Combinatorial Conf., pp. 239-254 (Utilitas Math. Pub. Co., Winnipeg).

P. Hall (1935), "On representatives of subsets", J. London Math. Soc. 10, 26-30.

Michael Mather (1976), "The Rugby footballers of Croam", J. Combinatorial Theory (Series B), 20, 62-63.

Guy H. J. Meredith and E. Keith Lloyd (1972), "The Hamiltonian graphs $0_{4}$ to $0_{2}$ ", Combinatorics (Institute of Mathematics and its Applications, Southend-on-Sea).

Guy H. J. Meredith and E. Keith Lloyd (1973), "The footballers of Croam", J. Combinatorial Theory (Series B), 15, 161-166.

Oystein Ore (1960), "Note on Hamilton circuits", American Math. Monthly, 67, 55.

Anne Penfold Street and W. D. Wallis (1977), Combinatorial Theory; An Introduction (C.B.R.C., Winnipeg).

Department of Mathematics

University of Newcastle

Newcastle, N.S.W. 2308 